\title{
Molecular dynamics simulations of silicon wafer bonding
}

\author{
D. Conrad, K. Scheerschmidt, U. Gösele \\ Max Planck Institute of Microstructure Physics, Weinberg 2, D-06120 Halle, Germany \\ (Fax: + 49 -345/5582-917, E-mail: CONRAD@ mpi-msp-halle.mpg.de)
}

Received: 25 July 1995/Accepted: 29 August 1995

\begin{abstract}
Molecular dynamics simulations based on a modified Stillinger-Weber potential are used to investigate the elementary steps of bonding two $\operatorname{Si}\left(\begin{array}{lll}0 & 0 & 1\end{array}\right)$ wafers. The energy dissipation and thus the dynamic bonding behaviour are controlled by the transfer rates for the kinetic energy. The applicability of the method is demonstrated by studying the interaction of perfect wafer surfaces (UHV conditions). First calculations covering the influence of surface steps, rotational misorientations and adsorbates are presented.
\end{abstract}

PACS: 02.70 . Ns; 34.20; 68.35

Silicon wafer bonding has become increasingly promising for silicon-on-insulator and micromechanical applications [1-4]. In most cases, silicon wafer bonding is performed under hydrophilic surface conditions in which a native (or purposely thermally grown) $\mathrm{SiO}_{2}$ layer on top of the silicon surface is covered with one or two monolayers of water $[3,4]$. Alternatively, hydrophobic silicon surfaces, which are mainly terminated by hydrogen and to a smaller extent by fluorine atoms, can be bonded $[5,6]$. Both hydrophilic and hydrophobic wafer bonding at room temperature lead to rather weak bonding energies compared to covalent bonding energies [3-6]. In order to reach high bonding energies, room-temperature bonded wafers have to be annealed at elevated temperatures.

The present paper will mainly deal with the question of whether two clean silicon surfaces (as obtained under appropriate ultrahigh vacuum conditions) can form covalent bonds across the interface already at room temperature. The problem is investigated in terms of Molecular Dynamics (MD) simulations. MD simulations have become a powerful tool for studying interface structures [7] as well as the mechanisms involved in gliding, friction, wear and adhesion [8] and the cleaving of crystals $[9,10]$. A first attempt to describe room-temperature wafer bonding of hydrophilic $\mathrm{SiO}_{2}$ surfaces was made by Garofalini [11]. The present study is probably the first attempt to deal with silicon itself.
We start with the simplest situation with respect to theory, viz. clean $\mathrm{Si}\left(\begin{array}{ll}0 & 0\end{array}\right)$ surfaces with or without inclusions of surface steps and adsorbates. First, in Sect. 1 we will derive a modified Stillinger-Weber potential which describes the $\operatorname{Si}\left(\begin{array}{lll}0 & 01\end{array}\right)$ surface better than the originally suggested potential [12] does. In Sect. 2 we will discuss how heat conduction processes can be included, which is essential to describe an energy transfer to a macroscopic substrate. Two different approaches will be pursued. In our study of the interaction of reconstructed surfaces, different initial situations will be investigated in Sect. 3, including two surfaces without misorientation, with a rotational misorientation of $90^{\circ}$ or of only a few degrees to simulate a twist boundary. Finally, the influence of surface steps and of fluorine-passivated surfaces will be simulated.

\section{Empirical potential}

The empirical potential proposed by Stillinger and Weber (SW) [12] is by far the most frequently used potential for MD studies of group IV semiconductors. Many bulk and defect properties have been described satisfactorily by this potential [13]. The potential has also been used for surface studies [14]. However, as mentioned by Balamane et al. [15], a serious shortcoming of the SW potential shows up in the treatment of the $\left(\begin{array}{lll}0 & 0 & 1\end{array}\right)$ surface. For the truncated bulk all contributions to the potential energy of the surface atoms are in equilibrium, resulting in vanishing forces. This feature is due to the general form of this potential together with its special chosen value of a cutoff radius rather than the special choice of parameters. Adding new terms can be useful to describe the surface properties more accurately [16], but it is also possible to improve the given potential qualitatively without changing the functional form. For diamond, Skokov et al. [17] changed the parameters in the original SW potential in such a way that they were able to simulate the $\mathrm{C}\left(\begin{array}{lll}0 & 0 & 1\end{array}\right)-2 \times 1$ surface reconstruction. This potential generates nearly instantaneously a fully dimerized diamond (0 01 1) surface starting from the truncated bulk geometry at room temperature. Recently, 
$\mathrm{ab}$ initio calculations confirmed such a behaviour for silicon [18], i.e. there is supposed to be no activation energy for a reconstruction. The important feature is the inclusion of second-nearest-neighbour interactions. The cutoff is chosen a little larger than the second-nearestneighbour distance so that these interactions do not play an essential role for nearly tetrahedral configurations.

Using empirical potentials, one should focus on properties common for similar materials. As a consequence, a simple change in energy- and length-scales should yield similar results for $\mathrm{C}, \mathrm{Si}$ and $\mathrm{Ge}$. Such a rescaled $\mathrm{SW}$ potential was successfully used to study the properties of amorphous germanium [19].

We rescaled the modified SW potential given by Skokov et al. [17] for diamond to appropriate energyand length-scales for silicon. According to Stillinger and Weber, the potential energy $V$ is expanded in two- and three-body contributions.

$V=\sum_{i<j} f_{2}\left(r_{i j}\right)+\sum_{i<j<k} f_{3}\left(r_{i j}, r_{i k}, r_{j k}\right)$

with

$f_{2}\left(r_{i j}\right)=A\left(B r_{i j}^{-p}-1\right) \exp \left(\frac{\lambda}{r_{i j}-a}\right)$

and

$f_{3}\left(r_{i j}, r_{i k}, r_{j k}\right)=h_{i j k}+h_{k i j}+h_{j k i}$,

$h_{i j k}=\lambda\left(\cos \theta_{j i k}+\frac{1}{3}\right)^{2} \exp \left(\frac{\delta}{r_{i j}-a}+\frac{\delta}{r_{i k}-a}\right)$

where $r_{i j}$ is the distance between atoms $i$ and $j$, and $\theta_{j i k}$ is the angle between vectors $r_{i j}$ and $r_{i k}$. Here, $r$ is expressed in dimensionless units where the unit distance is $\sigma=2.351 \AA$, i.e. the equilibrium nearest-neighbour-distance in the silicon crystal. Energies are measured in dimensionless units where the unit energy is $\varepsilon=2.1675 \mathrm{eV}$, i.e. the energy per bond in the silicon crystal. In addition, a dimensionless cutoff radius is introduced in such a way that all terms vanish for $r \geq a$. The other parameters are $A=$ 2.91411, $B=0.22530, p=4.0, a=1.7, \gamma=0.57, \lambda=6.0$, $\delta=0.66$ such that the cohesive energy and the lattice constant are accurately reproduced, whereas other properties, e.g. the melting point, certainly have changed. In the original SW potential for silicon, the parameters were $A=7.04956, \quad B=0.60222, \quad p=4.0, \quad a=1.8, \quad \gamma=1.0$, $\lambda=21.0, \delta=1.2$ with the unit of energy $\varepsilon=2.1675 \mathrm{eV}$ and the unit length $\sigma=2.0951 \AA$.

This modified SW potential leads to a symmetric dimerization of the surface within a few $10^{-13} \mathrm{~s}$. The dimer bond length is $2.4 \AA$ and the energy gain is $1.77 \mathrm{eV}$ per dimer $\left(0.96 \mathrm{~J} \mathrm{~m}^{-2}\right)$, in good agreement with both experiments and ab initio calculations [20]. Further quantitative improvements will be discussed elsewhere.

\section{Computational procedure}

In MD simulations the classical equations of motion are integrated. In our case the fifth-order Gear algorithm is used for the integration scheme. We extended the code that Garofalini and co-workers used in their study of glasses [21] using our interaction potential and the linked cell algorithm proposed by Grest et al. [22]. There are periodic boundary conditions in the directions parallel to the surface, whereas the whole system can move freely in the direction perpendicular to the surface. To calculate the surface properties, several runs with slabs of up to 12 atomic layers in depth were performed. The effect of the surface reconstruction turned out to be negligible in depths below the fourth atomic layer; hence the general behaviour is not affected if atoms on the opposite side are fixed. In order to be able to describe the relevant processes on the interacting surfaces, we had to use a rather short integration time step of $2.5 \times 10^{-16} \mathrm{~s}$.

Luedtke and Landman [23] pointed out that even a successive deposition of single atoms on a substrate can lead to the melting of the surface. This effect is much stronger in simulating wafer bonding, where many bonds are broken or created at the same time. Therefore, we introduced an energy transfer from the outermost atomic layers into a substrate to model a macroscopic heat conduction, which can hardly be incorporated directly as was pointed out already by Stillinger and Weber [24]. Two different approaches were investigated.

In the first approach, the velocities of the outermost atomic layers are rescaled to an average constant temperature of $300 \mathrm{~K}$, suggesting a situation in which the heat conduction takes place very fast. Therefore, we term this approach "fast heat conduction approach". It should be understood as a certain way of simulating macroscopic heat conduction, and, hence, the flow of kinetic energy away from the interface. This temperature control was applied every 100 time steps, where the kinetic energy was averaged over the 100 time-step intervals. A more frequent rescaling would influence the lattice vibrations.

In the second approach, to remove energy every 100 time steps we rescaled the kinetic energy of the same atoms as above by the constant factor of 0.98. Again, the kinetic energy was averaged over the 100 time steps. We termed this approach, which allows the layers below the surfaces to heat up, "slow heat conduction approach". This temperature control was finished when the kinetic energy of the atoms near the interface no longer changed significantly, i.e. when the process of creating new bonds had finished. The specific factor of 0.98 was chosen such that the bulk layers could not cool down below the initial temperature of $300 \mathrm{~K}$ during the simulation. For a somewhat smaller scaling factor, the temperature of the bulk layers will be constant, yielding the same results as in the first approach. The energy which was removed by the rescaling procedures corresponds to a flow of kinetic energy into a macroscopic substrate. Within the first approach, the average kinetic energy at the borders of the simulated system remains constant, whereas within the second approach the heat flow is chosen such that the temperature far away from the interface remains constant.

\section{Results and discussion}

In the following, we will describe the simulated wafer bonding processes for different initial conditions. Based 

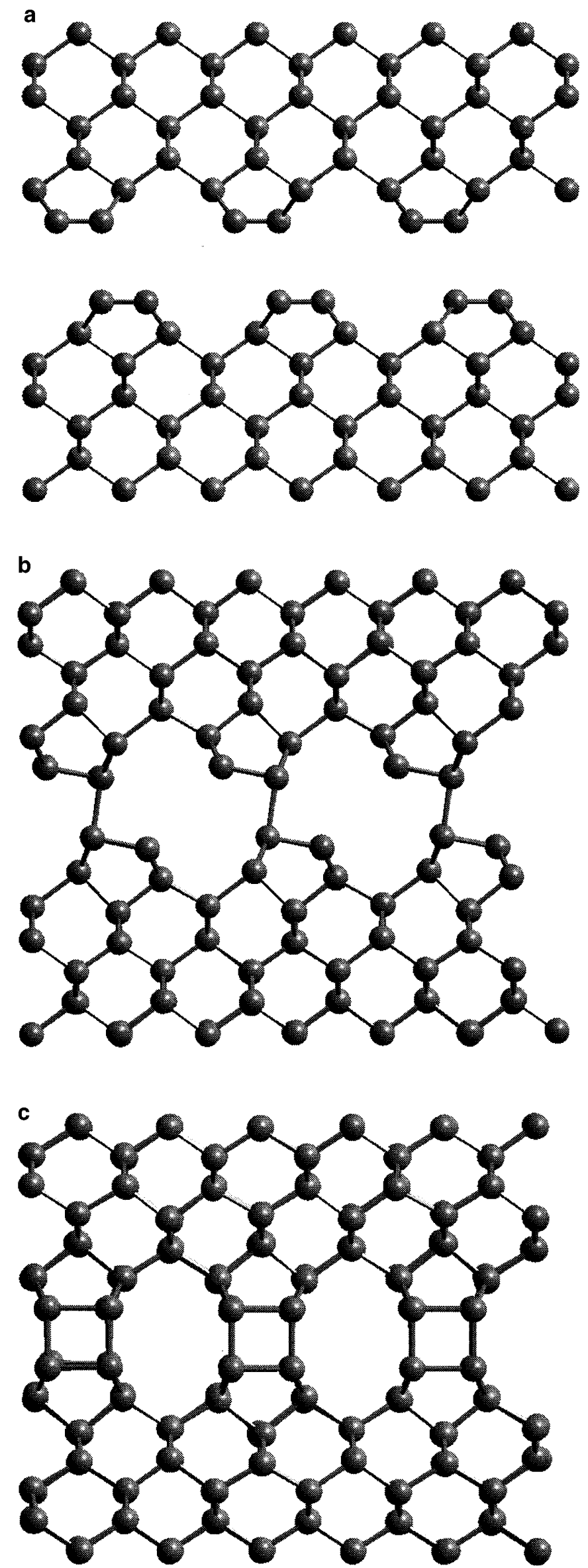

on the boundary conditions chosen, the two slabs can move perpendicular as well as parallel to the interface normal. The only geometrical parameter fixed is the twist angle of the two slabs.

\subsection{Perfect alignment (twist angle of $0^{\circ}$ )}

Figure 1 shows the bonding process for the perfectly reconstructed surfaces. The start is shown in Fig. 1a: two slabs of 12 atomic layers each (not all layers are shown in the figures) are assumed to be at a distance of $3.8 \AA$. The two opposite faces are reconstructed in a $2 \times 1$ pattern. The distance is measured with respect to the dimer planes. In the first example, within the fast heat conduction approach, energy is removed by rescaling the velocities of the five outermost atomic layers of each slab to a temperature of $300 \mathrm{~K}$. Because of the attractive forces, the slabs move towards each other and new "bonds" (with respect to the covalent radius) are forming (Fig. 1b). The simulation is performed for several $10^{4}$ time steps until the configuration no longer changes. Subsequently, the ensemble is slowly cooled down to $0 \mathrm{~K}$ to obtain the equilibrium atomic positions and energy. The final configuration, not corresponding to the perfect crystal, is shown in Fig. 1c. It is noteworthy that each interface atom has four next neighbours. The energy gain per interface area is $2.1 \mathrm{~J} \mathrm{~m}^{-2}$ with respect to the reconstructed surfaces.

In the second example, within the slow heat conduction approach, energy is removed by rescaling the velocities of the same atoms as in the first run by a constant factor. Starting with the configuration described above, the dynamics changes dramatically: the energy gained by lowering the distance of the slabs causes the dimers to break up. The surface atoms find their bulk positions yielding a perfect crystal. Figure 2 shows a snapshot taken at $2.5 \times 10^{-13} \mathrm{~s}$. At this point, the interface is nearly perfectly bonded. Continuation of the dynamics leads to the annealing of the imperfect regions which are still present. A perfectly bonded structure results with an energy gain of approximately $5.0 \mathrm{~J} \mathrm{~m}^{-2}$ per interface area.

\subsection{Rotational misorientation of $90^{\circ}$}

Figure 3 deals with a relative rigid rotation by $90^{\circ}$, because atomic height steps occur quite frequently on wafer surfaces. The other initial conditions are the same as in the previous case. In our simulations, the dimer bonds of only one surface break, depending on a relative shift of the surfaces. However, at elevated temperatures this behaviour may change. Here we give only the results of the room-temperature simulations. In the final structure

Fig. 1a-c. Snapshots (cross-section) of MD run for perfect aligned reconstructed surfaces: (a) initial configuration, (b) the configuration after $10^{-12} \mathrm{~s}$ using the fast heat conduction approach, and (c) the final configuration resulting from the fast heat conduction (only atoms near the interface are drawn) 


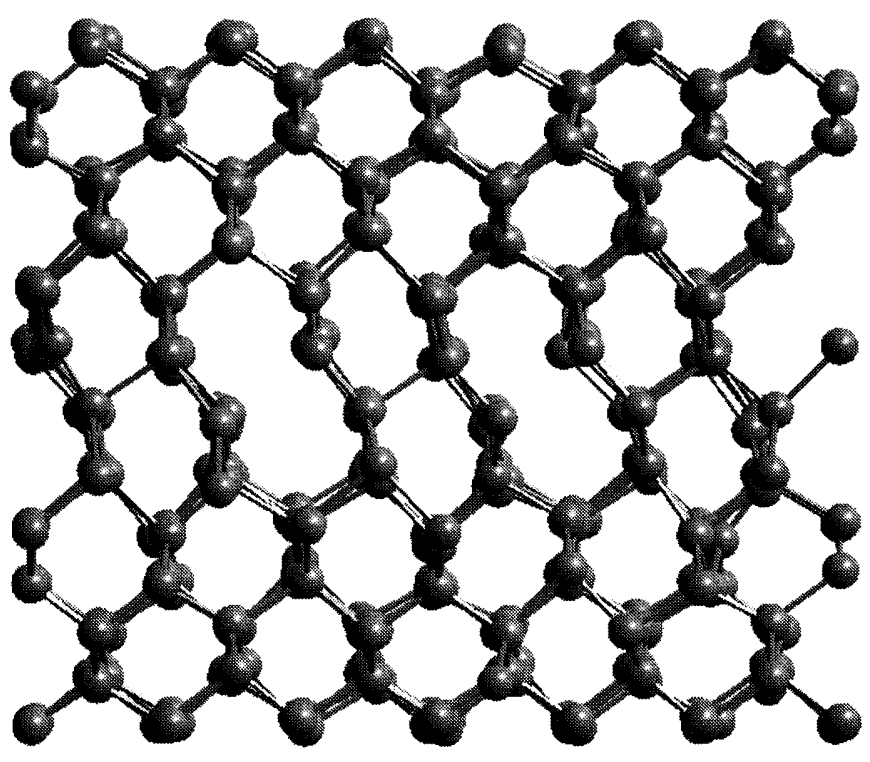

Fig. 2. Snapshot (cross-section) of MD simulation of perfect aligned surfaces under the slow heat conduction approach showing that new bonds have already been created, but the structure is still imperfect

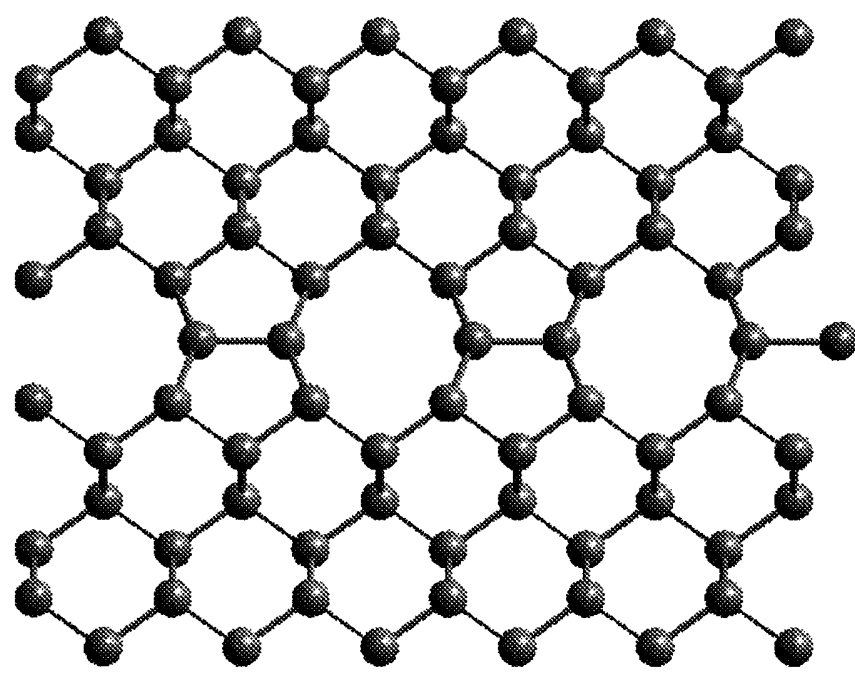

Fig. 3. Final configuration of bonded wafers with an initial rotation of $90^{\circ}$, cross-sectional view

(Fig. 3), all interface atoms are again fourfold-coordinated. The energy gain of this boundary is $2.5 \mathrm{~J} \mathrm{~m}^{-2}$ with respect to two reconstructed surfaces at an infinite distance.

\subsection{Small rotational misorientation}

To study the effect of a small twist angle, one surface is rotated by an angle of $4.58^{\circ}$, corresponding to a $\Sigma=313$ boundary. A plan view of the starting configuration is shown in Fig. 4a. Such a small twist angle may not occur in conventional growth processes, but the experimental wafer bonding equipment allows the combination of silicon wafers with any desired misorientation. When the surfaces closely approach, with bonds forming in between them (with respect to an appropriate cutoff radius), we observe three distinct regions on the surfaces (see Fig. 4b): one in which the dimer rows of the upper surface lie in between those of the lower one, another one in which the dimer rows of the surfaces lie on top of each other and a third one in which interface atoms have a bulk-like environment. These regions are divided by "interface dislocations". The simulation has been performed for $5 \times 10^{4}$ time steps at $300 \mathrm{~K}$. Probably, the crystalline regions will grow slowly during a long-time run. In order to accelerate the growth of the crystalline regions, the simulation is continued for $5 \times 10^{4}$ time steps at $900 \mathrm{~K}$ before it is cooled down again yielding an interface energy of $3.7 \mathrm{~J} \mathrm{~m}^{-2}$. Figure 4 shows a top view of the interface: (a) the initial configuration; (b) after $5 \times 10^{5}$ time steps at $300 \mathrm{~K}$; and (c) the result of the long-time run at $900 \mathrm{~K}$ and subsequent cooling to $0 \mathrm{~K}$.

\subsection{Stepped surfaces}

To model a situation more closely related to experiments, we investigate the effect of surface steps. Both opposite surfaces have a step of one atomic height, with the dimer bonds of the upper terrace perpendicular to the step (single step type A (SA), see e.g. [25]). The upper (lower) terraces are facing each other having a width of $23.04 \AA$ (7.68 $\AA$ ). Depending on the specific heat conduction approach, the behaviour of the upper terraces is similar to case (i). The behaviour of the lower terraces corresponds to the avalanche effect described by Nelson et al. [26] and is supposed to occur for any substrate thickness. The bonding of the lower terraces can be understood as follows. In the beginning, only the upper terraces interact and approach each other, inducing an elastic deformation at the step and hence forcing the lower-terrace atoms of one surface closer to those on the other surface. At a certain point, also the atoms on the lower terraces may interact and form a bonded interface. The energy gain owing to the creation of new covalent bonds is much higher than the energy necessary for a small elastic distortion of atoms from a tetrahedral configuration. Figure 5 shows a snapshot at $10^{-12} \mathrm{~s}$, when the lower terraces get into contact.

\subsection{Passivated surfaces}

The inclusion of adsorbates on the surfaces strongly influences the bonding properties. Using the SW potential for $\mathrm{Si}-\mathrm{F}$ interactions given by Stillinger and Weber [24], we simulated two surfaces passivated by fluorine. Starting with an initial separation of $5 \AA$ with respect to the dimer bonds the simulation predicts that the repulsive forces of the F-atoms dominate and prevent bonding. Recent experiments support this prediction [27,28].

\section{Summary}

We investigated various elementary steps of the silicon wafer bonding process by molecular dynamics simula- 


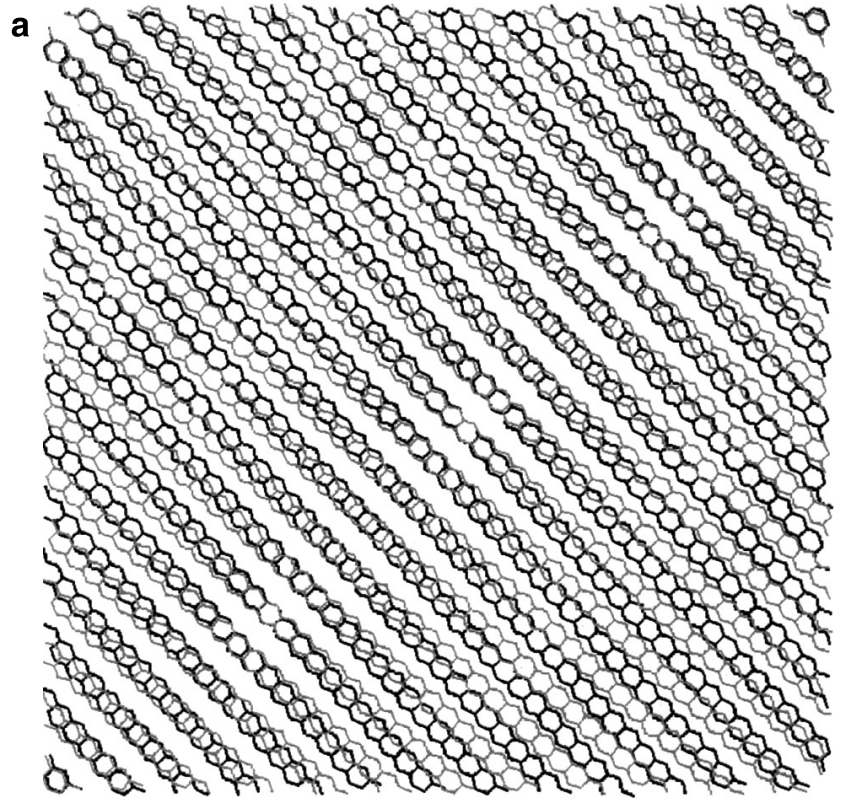

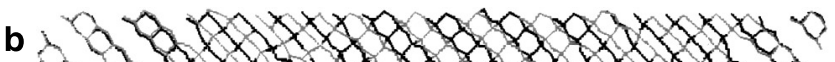

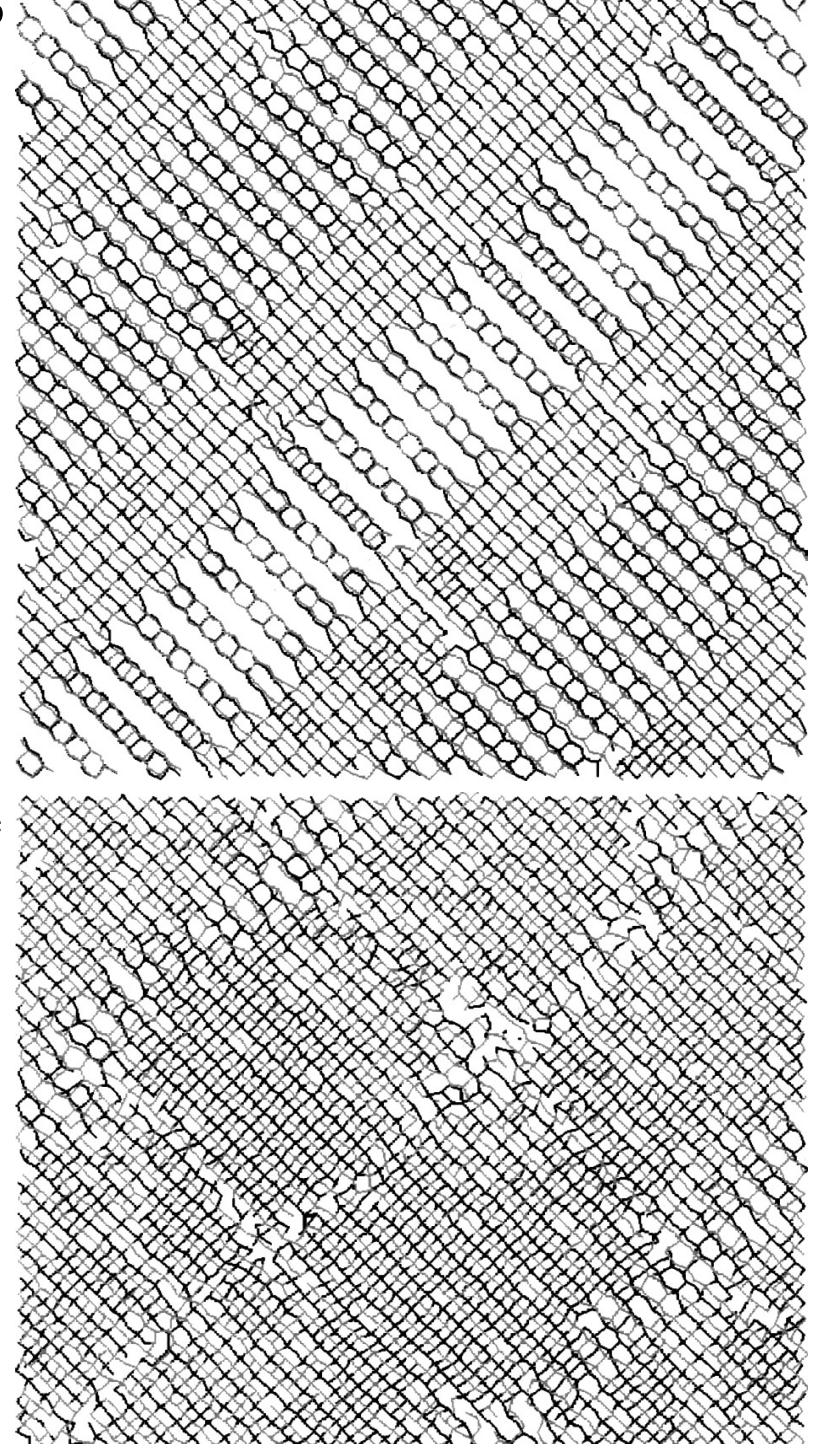

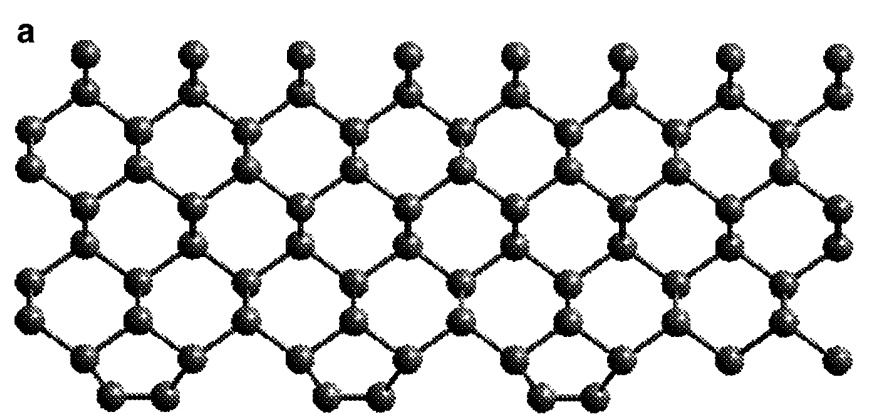
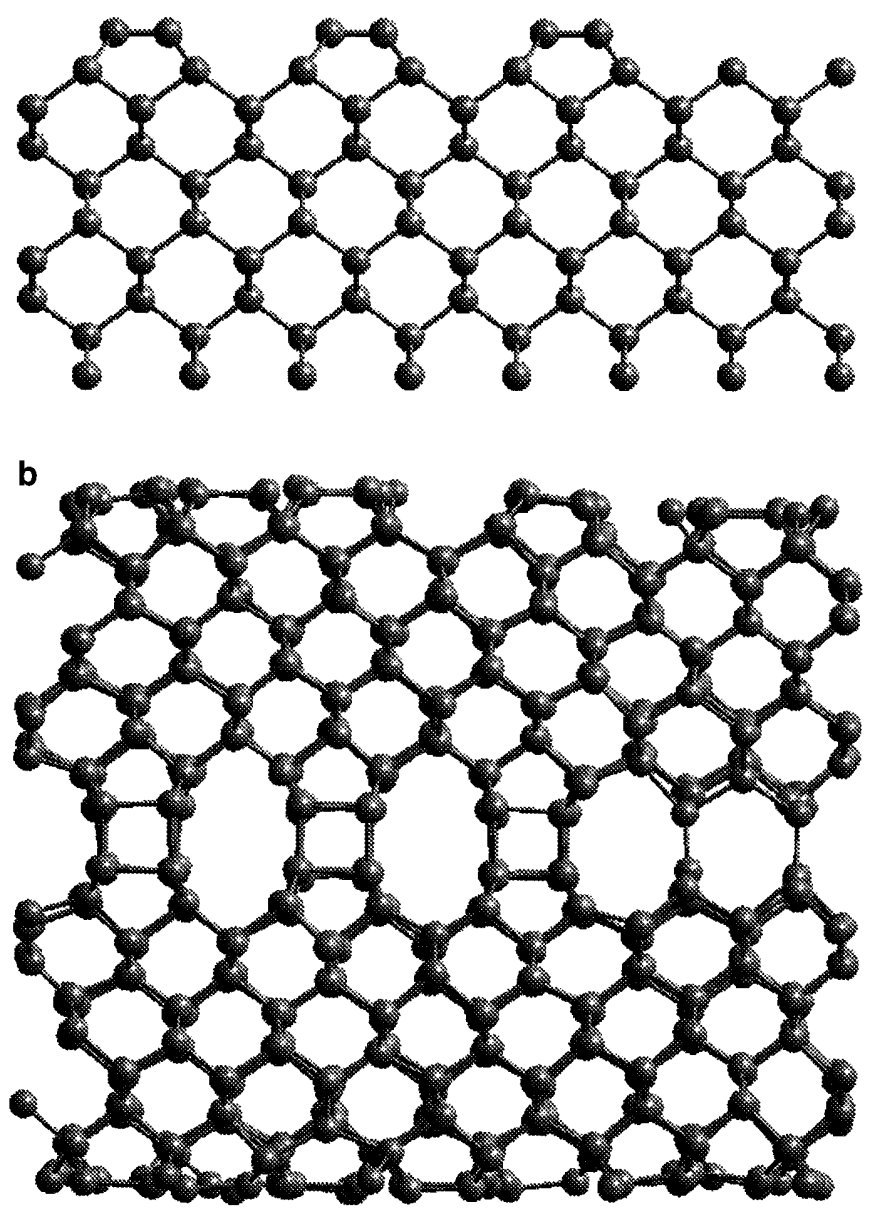

Fig. 5a,b. Simulation of $\operatorname{Si}(001)$ wafer bonding including oneatomic-height steps. (a) Starting configuration showing the surface steps, (b) snapshot of a cross-section at $10^{-12} \mathrm{~s}$ showing the contacting of the lower terraces

Fig. 4a-c. Three stages of the simulation for the bonding of wafers rotationally misoriented by $4.58^{\circ}$. The figures show a plan view on the interface, the light ( dark) grey lines are the bonds of two atomic layers of the upper (lower) surface. In (a) the starting configuration, in (b) the configuration after $5 \times 10^{4}$ steps at $300 \mathrm{~K}$, and in (c) the situation after $5 \times 10^{4}$ time steps at $900 \mathrm{~K}$ and subsequent cooling down 
tions. For this purpose, we modified the original Stillinger-Weber potential for silicon to improve the description of the spontaneous reconstruction of the Si $(001)$ surface. The qualitative change is the inclusion of second-nearestneighbour interactions. Energies and bond lengths of the reconstructed surface are in good agreement with both experimental and theoretical results. The interactions of flat Si (llll 01 ) $-2 \times 1$ surfaces were studied under different initial and boundary conditions. Our molecular dynamics simulations presented in examples (i)-(iv) predict that covalent bonding of clean and flat silicon surfaces should be possible already at room temperature. The detailed interface structures predicted by our simulations not only depend on the starting configuration of the two wafers (misorientation, steps) but to a certain extent also on how the generated heat is removed. The influence of surface steps and adsorbates is discussed qualitatively. Even with surface steps or some rotational misorientations present, the simulations predict that covalent bonding may be achieved by room-temperature bonding of elemental $\mathrm{Si}(001)$. Preliminary experimental results on room-temperature bonding of silicon wafers in ultrahigh vacuum

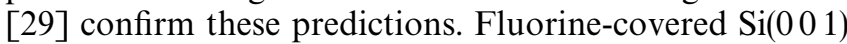
surfaces are predicted not to bond. A detailed study of the influence of the process parameters and the surface morphology will be given in a forthcoming paper.

Since the predictions of the molecular dynamics simulations appear to agree with experimental results, MD simulations are promising for developing the chemistry of appropriate "designer surfaces" with desirable bonding properties.

Acknowledgements. D.C. and K.S. would like to thank Peter Gumbsch for helpful discussions.

\section{References}

1. H. Baumgart, C. Hunt, M. Schmidt, T. Abe (eds.) : Proc. 2nd Int'l Symp. on Semiconductor Wafer Bonding: Science, Technology and Applications II (Electrochem. Soc., Pennington, NJ 1993)
2. C. Hunt, H. Baumgart, S. Iyer, U. Gösele, T. Abe (eds.) : Proc. 3rd Int'l Symp. on Semiconductor Wafer Bonding: Science, Technology and Applications (Electrochem. Soc., Pennington, NJ 1995)

3. S. Bengtsson: J. Electron. Mater. 21, 669 (1992)

4. Q.-Y. Tong, U. Gösele: Mater. Chem. Phys. 37, 101 (1994)

5. K. Ljungberg, A. Söderbärg, Y. Bäcklund: Appl. Phys. Lett. 62 , $1362(1993)$

6. Q.-Y. Tong, E. Schmidt, M. Reiche, U. Gösele: Appl. Phys. Lett. 64, 625 (1994)

7. K. Scheerschmidt, S. Ruvimov, P. Werner, A. Höppner, J. Heydenreich: J. Microsc. 179, 214 (1995)

8. J.R. Smith, G. Bozzolo, A. Banerjea, J. Ferrante: Phys. Rev. Lett. 63, 1269 (1989)

9. J.C.H. Spence, Y.M. Huang, O. Sankey: Acta Metall. Mater. 41, $2825(1993)$

10. Y.M. Huang, J.C.H. Spence, O. Sankey, G.B. Adams: Surf. Sci. 256, 344 (1991)

11. S. Garofalini: In [1], 93-29, 57 (1993)

12. F.H. Stillinger, T.A. Weber: Phys. Rev. B 31, 5262 (1985)

13. M. Kohyama, S. Takeda: Phys. Rev. B 51, 13111 (1995)

14. Z. Zhang, H. Metiu: Phys. Rev. B 48, 8166 (1993)

15. H. Balamane, T. Halicioglu, W.A. Tiller: Phys. Rev. B 46, 2250 (1992)

16. C.P. Toh, C.K. Ong: Surf. Sci. Lett. 303, L348 (1994)

17. S. Skokov, C.S. Carmer, B. Weiner, M. Frenklach: Phys. Rev. B 49, 5662 (1994)

18. A.I. Shkrebtii, R. Di Felice, C.M. Bertoni, R. Del Sole: Phys. Rev. B 51, 11201 (1995)

19. K. Ding, C. Andersen: Phys. Rev. B 34, 6987 (1986)

20. P. Krüger, J. Pollmann: Phys. Rev. Lett. 74, 1155 (1995)

21. S. Garofalini: J. Non-Cryst. Solids 120, 1 (1990)

22. G.S. Grest, B. Dünweg, K. Kremer: Comp. Phys. Commun. 55, 269 (1989)

23. W.D. Luedtke, U. Landmann: Phys. Rev. B 40, 11733 (1989)

24. F.H. Stillinger, T.A. Weber: Phys. Rev. Lett. 62, 2144 (1989)

25. T.W. Poon, S. Yip, P.S. Ho, F.F. Abraham: Phys. Rev. B 45, $3521(1992)$

26. J.S. Nelson, B.W. Dodson, P.A. Taylor: Phys. Rev. B 45, 4439 (1992)

27. K. Ljungberg, H. Jansson, S. Bengtsson, A. Söderbärg: In [2] (1995) (in press)

28. K. Ljungberg: Private communication

29. U. Gösele, H. Stenzel: In [2] (1995) (in press) 